\title{
Una Articulación Teórica Entre Competencia Algebraica, Proceso De Algebrización Y Modelización Algebraica
}

\author{
Uma Articulação Teórica Entre Competência Algébrica, Processo De \\ Algebrização E Modelização
}

Maria Jose Ferreira da Silva

Pontifícia Universidade Católica de São Paulo - (PUC-SP)

Cecilia Gaita

Pontificia Universidad católica del Perú - (PUCP)

Saddo Ag Almouloud

Pontifícia Universidade Católica de São Paulo - (PUC-SP)

\begin{abstract}
Resumen
Este artículo, de corte teórico, tiene por objetivo articular tres enfoques del álgebra escolar que son: el desarrollo de la competencia algebraica, el proceso de algebrización y el de modelización algebraica. Tal articulación ha sido elaborada a partir del referencial en el que se basa la investigación (todavía en desarrollo) que trata de la formación continua de profesores, realizada en colaboración entre los grupos de investigación PEA-MAT de la PUC-SP y DIMAT de la PUCP, respectivamente, en el Proyecto Procesos de Enseñanza y Aprendizaje del Álgebra. Confirmamos la posibilidad de dicha articulación, la cual permite analizar con mayor detalle la enseñanza del álgebra escolar y verificar las ausencias, escasez y exceso de tareas que conducen al desarrollo de la competencia algebraica en el estudiante. Dicha articulación permite también construir organizaciones matemáticas y didácticas más completas para esa misma finalidad.
\end{abstract}

Palabras-clave: Competencia algebraica. Modelización algebraica. Proceso de algebrización.

\section{Resumo}

Este artigo, de cunho teórico, tem por objetivo realizar um ensaio de articulação entre três enfoques a respeito da álgebra escolar que são: o desenvolvimento da competência algébrica, o processo de algebrização e a modelização. Tal articulação foi construída a partir do referencial que embasou a pesquisa (ainda em andamento) que trata de formação continuada de professores realizada em colaboração pelos grupos de pesquisa PEA-MAT da PUC-SP e DIMAT da PUC-Peru, respectivamente, no Projeto Processos de Ensino e de Aprendizagem de Álgebra. Verificamos a possibilidade dessa articulação e ainda que ela nos permite analisar com mais clareza o ensino da álgebra escolar e verificar os pontos de escassez ou de excesso de tarefas que conduzam ao desenvolvimento da competência algébrica no aluno. Permite também construir organizações matemáticas e didáticas mais completas para o mesmo fim.

Palavras-chave: Competência Algébrica. Modelização Algébrica. Processo de Algebrização. 


\section{Introducción}

Este artículo presenta reflexiones originadas en el marco de un proyecto de investigación centrado en las interacciones entre investigadores de la PUC-SP y PUCP, en relación con los procesos de enseñanza y aprendizaje de la matemática en ambientes tecnológicos. Dicha iniciativa es desarrollada en forma colaborativa por los grupos de investigación Processos de Ensino e de Aprendizagem de Matemática - PEA-MAT, de la PUCSP, y Didáctica de las Matemáticas - DIMAT, que pertenece al Instituto de Investigación sobre la Enseñanza de las Matemáticas (IREM) de la PUCP. Tiene como objetivo analizar, tanto desde un punto teórico como práctico, cuestiones relativas a la complejidad de la inclusión de herramientas tecnológicas para la enseñanza y el aprendizaje de la Matemática no solo en la Educación Básica sino también en la Universidad.

Aunque inicialmente dicho proyecto tenía como foco la inclusión de herramientas tecnológicas, se hizo necesario realizar una reflexión teórica más profunda que justificara la inclusión de la tecnología en la enseñanza del álgebra. Así, se propone una primera articulación de tres enfoques respecto del álgebra escolar, el desarrollo de la competencia algebraica, el proceso de algebrización y la modelización algebraica.

\section{Revisión De La Literatura}

Se han realizado diversos estudios sobre la enseñanza y aprendizaje del álgebra en la Educación Básica; sin embargo, en este trabajo, consideraremos en particular los realizados por Chevallard y algunos de sus seguidores. A partir de la revisión de trabajos previos, coincidimos con Munzón, Bosch y Gascón (2015) cuando afirman que gran parte de las investigaciones en Didáctica de la Matemática que tratan del álgebra elemental se centran en el estudio de las dificultades de los estudiantes al inicio de su aprendizaje y de las posibles actuaciones del profesor durante la enseñanza para minimizarlas. Siguiendo a los autores, es mucho más difícil encontrar trabajos que estudien lo que se enseña con el nombre de álgebra elemental y, como consecuencia, lo que se entiende por álgebra en las clases de Matemáticas en la escuela.

Dichas afirmaciones parten de los trabajos de Chevallard (1984, 1989, 1990) y Gascón (1993, 1994, 1999) que reportan que el modelo dominante para el álgebra en la institución escolar es el de aritmética generalizada, la cual es construida a partir de un contexto numérico en el que las expresiones algebraicas surgen de la necesidad de representar y manipular números 
desconocidos a partir de datos que son conocidos. En ese contexto, la manipulación algebraica se reduce al cálculo algebraico como una prolongación del cálculo aritmético en el que la única diferencia es la representación de números por letras; esto se evidencia cuando se inicia la enseñanza del álgebra a través de la resolución de problemas aritméticos empleando ecuaciones.

Para Chevallard (1989, p.65), la funcionalidad del cálculo algebraico debe ser vista “como una perspectiva de renovación curricular que debe considerar, desde muy temprano, la utilización de parámetros en la producción y exploración de fórmulas para conducir a la noción de función”. El autor señala como la principal causa de las dificultades presentadas, tanto para la enseñanza, como para el aprendizaje del álgebra escolar al abandono del empleo de parámetros para la obtención de fórmulas y la noción de función. Para él, “el empleo de parámetros ocupa un papel central, desde el nivel más elemental de los estudios matemáticos, la noción de fórmula [...] directamente relacionada con la noción de función” (p.65). Sugiere, entonces, la modelización algebraica (que será definida posteriormente) como un proceso que debe permear toda la enseñanza del álgebra en la escolaridad básica, pues involucra la actividad de simbolización con el uso de letras para designar cantidades desconocidas, pero también incluye a los parámetros para el estudio de soluciones generales que implican el uso metódico de sistemas de representación que articulen varios registros semióticos. ${ }^{1}$

En esa misma línea, hemos identificado tal postura en algunos textos antiguos. Por ejemplo, en Sangiorgi (1959), encontramos discusiones respecto de la resolución de problemas, que involucran sistemas de ecuaciones lineales y en las que se propone la generalización de problemas. Luego, el autor del texto define lo que se entiende por problemas de primer grado y presenta las siguientes fases para su resolución: "convertir el problema en una ecuación; resolver la ecuación o el sistema de ecuaciones y discutir las soluciones" (p.151). En los procesos de formación continua que hemos implementado, en nuestro proyecto, hemos observado que los profesores siguen ese proceso, pero difícilmente discuten las soluciones. Posteriormente, el autor señala lo que entiende por generalización de un problema: "generalizar un problema es resolverlo de modo general, es decir, consiste en determinar una o más fórmulas que permitan resolver todos los problemas semejantes al considerado inicialmente. Con esa finalidad, es necesario usar letras en lugar de datos numéricos" (p. 153). Sin embargo, solo los pasos de resolución se pueden encontrar en textos actuales, la generalización de problemas fue abandonada, y solo aparece para resolver problemas de generalización de patrones. En el trabajo

\footnotetext{
${ }^{1}$ Un registro de representación es, según Duval (1999 apud ALMOULOUD, 2007, p.71), un sistema semiótico que tiene las funciones cognitivas fundamentales de objetivación, tratamiento y conversión, a nivel del funcionamiento cognitivo consciente.
} 
que hemos desarrollado con profesores en formación continua se pudo en evidencia que, cuando se les presentaban varios problemas con la misma estructura, ellos los resuelven de manera particular y no reconocen las semejanzas que existen.

Al respecto, Gascón (1994) propone considerar al álgebra como un medio de resolución de problemas, que se caracteriza por el estudio de un campo de problemas que contiene, no solamente problemas aritméticos, sino también problemas de construcciones geométricas, problemas de conteo simple, etc. Tales problemas, resueltos por métodos algebraicos, pueden proporcionar una representación global de las relaciones entre los datos e incógnitas, simbolizadas por letras que pueden asumir estatus diferentes (incógnita, número generalizado, variable), además del uso de parámetros, que conducirá a un modelo algebraico.

Dichos estudios fueron profundizados en otros trabajos como el de Bolea, Bosch y Gascón (1998) respecto al papel de la algebrización en el estudio de una organización matemática, como el del (2001), de los mismos autores, en donde se aborda el proceso de modelización algebraica y como el de Grugeon (2000), respecto a la competencia algebraica (definiremos más adelante lo que dicha autora entiende por competencia algebraica). Continuando con esas discusiones, Munzón, Bosch y Gascón (2010(a), (b), 2011, 2015) parten de un modelo epistemológico de referencia ${ }^{2}$, para el álgebra elemental, como instrumento y determinan etapas de algebrización y de modelización. Por otro lado, Grugeon (2000) señala que los conocimientos estructurados, desde la perspectiva de la Herramienta-Objeto, y la introducción al álgebra supone una ruptura epistemológica con la aritmética, que exige el desarrollo de una capacidad de adaptación para interpretar expresiones algebraicas, además de la capacidad de reconocer el papel del álgebra para expresar soluciones generales y demostrar propiedades numéricas.

Las discusiones iniciales, respecto de la enseñanza del álgebra escolar, en una formación continua de profesores de Enseñanza Básica, nos conducirán al tema de sistemas de ecuaciones lineales, utilizado como herramienta para la resolución de problemas y, en consecuencia, para determinadas cuestiones: ¿cuál es el papel de los sistemas de ecuaciones lineales en el desarrollo de la competencia algebraica? Para dar respuesta a ello, revisamos el trabajo de Coulange (2000), en donde se señala que gran parte de los problemas que son resueltos a través de sistemas de ecuaciones lineales presentan informaciones que no son redundantes ni

\footnotetext{
${ }^{2}$ Barquero, Bosch y Gascón (2013) indican que la dimensión epistemológica es importante y está presente en todo y cualquier problema didáctico, pues a través de ella buscamos entender: la amplitud del ámbito matemático en el que se sitúa el problema didáctico; los tipos de problemas provenientes de la enseñanza y del aprendizaje a partir de la problemática; los intentos de abordar e incluso solucionar tal problemática; cuáles son las razones de ser de ese objeto matemático y de la problemática de su enseñanza.
} 
contradictorias, lo que conduce a un trabajo mecánico de resolución del sistema. Esa afirmación fue constatada a través de las discusiones sostenidas con los profesores en formación continua y también con el análisis de libros didácticos brasileros. En ese contexto, surge la propuesta de articular el desarrollo de la competencia algebraica, siguiendo a Grugeon (1995, 2000), los procesos de algebrización y de modelización, desarrollados por Munzón, Bosch y Gascón (2010(a), (b), 2011, 2015), a partir de los resultados de Chevallard (1984, 1989, 1990).

Para desarrollar la noción de competencia algebraica, Grugeon se basa en la Teoría de Registros de Representación Semiótica -TRRS (Duval, 1995) y en la Dialéctica Herramienta-Objeto y la noción de marco (Douady, 1986) de las cuales presentaremos, a continuación, un breve resumen

Duval (1999) afirma que un registro remite a la dimensión semiótica, imprescindible para la comprensión y representación de los objetos matemáticos. Para el autor, un registro de representación es un sistema semiótico que tiene las funciones cognitivas fundamentales de formación, tratamiento y conversión, en el nivel del funcionamiento cognitivo consciente. Esa noción proporciona al profesor un medio para hacer más accesible la comprensión de nociones matemáticas, enfatizando en la importancia del cambio de registros y de una coordinación entre ellos. Un cambio de registro presenta ventajas desde el punto de vista del tratamiento y puede facilitar la comprensión o descubrimiento, por lo que es necesario distinguir dos tipos heterogéneos de transformaciones de las representaciones: el tratamiento y la conversión. De acuerdo con Duval:

Un tratamiento es una transformación de una representación en otra del mismo registro, esto es, una transformación estrictamente interna a un registro. Existen tratamientos que son específicos a cada registro y que no requieren de ninguna contribución externa para ser realizados o justificados. Una conversión es una transformación de una representación de un registro $\mathrm{D}$ en otra representación de un registro A, conservando, al menos, una referencia al mismo objeto o a la situación original, pero cambiando, de hecho, el contenido de la representación. . (DUVAL, 1999, p. 30, Apud Almouloud, 2007, p.72).

En relación a la Dialéctica herramienta-objeto, Douady define los constructos "objeto" y "herramienta" de la siguiente forma:

Un concepto es herramienta cuando nos interesamos por su utilización para resolver un problema. Una misma herramienta podrá ser adaptada para diferentes problemas. Por objeto, entendemos el objeto cultural situado en un edificio más amplio, que es el saber sabio en un determinado momento reconocido socialmente. (DOUADY, 1986, p. 9, Apud Almouloud, 2007, p.62).

Para Douady (1993, p. 389, Apud Almouloud, 2007, p.64), un marco “está constituido de herramientas de una parte de la matemática, de las relaciones entre objetos, de formulaciones 
eventualmente diferentes y de imágenes mentales asociadas a herramientas y relaciones". Así, un cambio de marco es un medio para obtener formulaciones diferentes de un problema, que permite abordar las dificultades encontradas desde una perspectiva distinta, ofreciendo herramientas y técnicas que no se contemplan en una primera formulación. Mientras que los juegos de marcos son cambios de marcos provocados por iniciativa del docente, cuando selecciona problemas adecuados para hacer progresar las fases de investigación y evolucionar las concepciones de los alumnos.

De otro lado, los procesos de algebrización y modelización se basan en la Teoría Antropológica de lo Didáctico (TAD) que estudia las relaciones entre sujeto-institución -saber. Para Chevallard (1992), la TAD tiene por objetivo el estudio del hombre frente al saber matemático, más específicamente, frente a situaciones matemáticas. Para él (ídem, 1999) el saber matemático organiza una forma particular de conocimiento, producto de la acción humana en una institución caracterizada por cualquier cosa que se produzca, se utilice y se enseñe, además de poder eventualmente transponer las instituciones.

Para Chevallard (1999) las nociones de (tipos de) tarea, (tipo de) técnica (manera de hacer), tecnología (justificación de la técnica) y teoría (campo de saberes científicos que justifican la tecnología) permiten modelizar las prácticas sociales en general y, en particular, la actividad matemática. La necesidad de reconstruir tareas, en tanto construcciones institucionales, caracteriza un problema que debe ser resuelto dentro de la propia institución y que, en el caso del salón de clases, por ejemplo, es una cuestión didáctica. Un conjunto de técnicas, de tecnologías, y de teorías organizadas para un tipo de tarea forma una "praxeología" puntual y se refiere al hecho de que una práctica humana, dentro de una institución, siempre está acompañada de un discurso, más o menos desarrollado, de un logos, que la justifica, la acompaña y que es su razón de ser. Una praxeología que integra varias praxeologías puntuales, cuyas técnicas son justificadas por un mismo discurso tecnológico se constituye en una praxeología local.

Un saber, en esa teoría, se refiere a una praxeología u organización particular que le permite funcionar como una máquina de producción de conocimiento. La praxeología asociada a un saber es la unión de do bloques: saber hacer (técnico/práctico) y saber (tecnológico /teórico) cuya ecología se refiere a las condiciones de su construcción y vida en las instituciones que la producen, utilizan o transponen. Siguiendo a Chevallard (1999), las praxeologías (u organizaciones) asociadas a un saber matemático son de dos tipos: matemáticas y didácticas. Las organizaciones matemáticas se refieren a la realidad matemática que se puede construir 
para ser desarrollada en una sala de clase y las organizaciones didácticas se refieren a las maneras de hacer esa construcción.

En un proceso de formación de saberes/conocimientos, las praxeologías envejecen, pues sus componentes teóricos y tecnológicos pierden crédito. Continuamente, en una determinada institución I surgen nuevas praxeologías que podrían ser producidas o reproducidas si existen en alguna institución I’. El paso de la praxeología de la institución I a la institución I' es llamado por Chevallard (2002) Transposición, más precisamente, Transposición Didáctica cuando la institución de destino es una institución de enseñanza (escuela, clase, etc.)

Los constructos teóricos propios de la TAD (praxeologías), de la TRRS (registro de representación semiótica, conversión y tratamiento), y las nociones de herramienta, objeto y marco desempeñarán un papel importante en el análisis de los tres aspectos aquí analizados: el desarrollo de la competencia algebraica, el proceso de algebrización y el de modelización algebraica, así como en el intento por articularlos. Ellos permitirán explicitar características importantes del álgebra escolar, tales como: la capacidad de cambiar de punto de vista, de traducir un problema de un marco a otro, la conversión de representaciones de un registro a representaciones en otro registro, con la finalidad de movilizar otras herramientas para dar soluciones diferentes a las consideradas inicialmente. Mientras que los sistemas de ecuaciones lineales serán herramientas en la medida que permitan resolver, y también serán vistos como objetos cuando se contemple, por ejemplo, el uso de parámetros.

\section{Elementos Teóricos}

Así, en el siguiente apartado presentaremos lo que caracteriza el desarrollo de la competencia algebraica, seguido de una explicación sobre en qué consisten los procesos de algebrización y modelización, para luego proponer una articulación de estos elementos de modo que puedan ser empleados en futuras formaciones de profesores y trabajos de investigación.

\subsection{La competencia Algebraica}

A partir de la síntesis de trabajos en Didáctica de la Matemática, Grugeon (2000) presenta algunos aspectos para el desarrollo de una competencia algebraica. En cuanto a la estructuración de los conocimientos algebraicos según las dimensiones herramienta y objeto, la autora afirma que es necesario considerar la capacidad de interpretar y construir expresiones 
y relaciones algebraicas para traducir un problema, además de movilizar herramientas algebraicas para la resolución de tareas del dominio del álgebra que implican generalización y prueba, modelización, aritmética tradicional, etc.

La dimensión herramienta del álgebra se moviliza en diferentes contextos y dominios de empleo tales como tareas de resolución, de prueba, siendo las que se desarrollan en la "aritmética tradicional" solo algunas de ellas. Como objeto, es necesario considerar el estatus de los objetos del álgebra, así como los dos aspectos: el sintáctico (interpretación) y el semántico (significado) de las expresiones algebraicas para manipularlas formalmente y para entonces considerar la dimensión técnica del tratamiento algebraico. Para la autora "la significación de una expresión algebraica reside, a su vez, en su sintaxis, su denotación, su interpretación en relación con los marcos matemáticos en juego y sus significados" (ibid., p.9). Así, la competencia algebraica se evalúa por medio de las capacidades técnicas de orden sintáctico y capacidades interpretativas, así como de dar sentido a las expresiones.

La autora desarrolló una estructura de análisis que, del lado del alumno, permite analizar su producción y verificar su funcionamiento en álgebra elemental y, desde la institución, relaciona ese funcionamiento con la enseñanza recibida. Esta estructura está apoyada en tres aspectos principales: una ruptura epistemológica entre aritmética y álgebra, una introducción de nuevos objetos en álgebra y la consideración de campos de problemas algebraicos.

La ruptura epistemológica con la aritmética es necesaria pues, de acuerdo con Chevallard (1989 citado en Grugeon (2000, p.3), "la herramienta esencial de la aritmética es el lenguaje ordinario y el cálculo con los números [...]. Ella es esencialmente un saber oral que sólo confía en el lápiz y papel para la realización de las operaciones con los números". Mientras que la introducción de "nuevos" objetos del álgebra, en general, se realiza a partir de la resolución de problemas que conducen a la representación de una ecuación y, a continuación, a su resolución. Sin embargo, siendo uno de los objetos del álgebra la utilización de letras, la autora advierte que en aritmética ellas pueden ser sólo una etiqueta y da como ejemplo la expresión $12 \mathrm{~m}$ que podría ser empleada tanto para representar 12 metros, como 12 motos. Eso explicaría por qué en estudios sobre los errores y dificultades de los alumnos en relación al álgebra realizados por Grugeon (2000) se encontró que los estudiantes consideraban que las letras podían no ser utilizadas en los cálculos, o que podían ser sustituidas por un valor numérico en algunos cálculos, o que podían ser incógnitas para designar números desconocidos, o que podían representar números generalizados cuando asumían varios valores e, incluso, podían ser variables en un contexto funcional. 
Otros objetos del álgebra serán las expresiones algebraicas que deben ser construidas por medio de una representación formal de problemas a partir de números y signos operatorios con sentidos diferentes de los utilizados en aritmética. En dichas expresiones algebraicas, existe un cálculo a efectuar y ellas mismas pueden ser un resultado. Grugeon (2000) afirma que un cálculo algebraico puede, por ejemplo, resultar en una expresión del tipo $x+3$ Una misma expresión algebraica puede representar uno o varios objetos o procesos operativos "lo que hace que la actividad matemática sea eficaz". Por ejemplo, la expresión $3 x+1$ puede interpretarse como:

- la operación correspondiente a tomar un número, multiplicarlo por 3 y añadirle 1 ;

- o la expresión obtenida partir del número $x$, expresión que resulta del proceso operatorio como un todo;

- o la expresión genérica de un número congruente a 1 módulo 3;

- o la imagen de $x$ a través de la función de variable $x$, que asocia a cada $x$, $3 x+1$

- o una cadena de símbolos que no representa nada, pero que se puede combinar con otras expresiones utilizando reglas bien definidas. (GRUGEON, 2000, p.6)

Las ecuaciones surgen a partir de problemas numéricos que conducen a su escritura y luego a su resolución. Para la autora

el aprendizaje del álgebra no se limita al tratamiento de expresiones algebraicas, aunque esta dimensión sea la más evocada en los trabajos. La competencia algebraica no puede ser evaluada sólo por la capacidad de transformar expresiones, también debe serlo por la capacidad de resolver problemas en los que el álgebra interviene como herramienta pertinente. Es capaz de producir expresiones y relaciones para traducir un problema, de interpretar y luego movilizar las herramientas algebraicas adaptadas para su resolución. (GRUGEON, 2000, p.7)

Añade además que las ecuaciones en las que la incógnita está sólo en un miembro pueden ser resueltas por adaptación de métodos aritméticos, pero las que presentan la incógnita en los dos miembros conservan la igualdad (equivalencia) y provocan una ruptura con los métodos anteriores.

Además de lo anterior, la autora asegura que el aprendizaje del álgebra puede ser evaluado por la capacidad para resolver problemas a partir de la producción de expresiones y relaciones algebraicas que los traducen y de sus interpretaciones para entonces utilizar herramientas algebraicas que se adaptan a su resolución.

En ese sentido, la autora caracteriza los problemas algebraicos en aritméticos, de generalización y de modelización. La introducción al pensamiento algebraico se garantiza a través de problemas aritméticos clásicos, lo que caracteriza la aritmética generalizada. Sin 
embargo, esto no es suficiente para que los alumnos construyan significados para las nuevas expresiones; se hace necesario considerar otros tipos de problemas. Los problemas de generalización, a su vez, permiten expresar y justificar relaciones numéricas generales, que serán enunciadas como propiedades, pero también exigen recurrir a nuevas relaciones. Un ejemplo de este tipo de problema es presentado por Chevallard (1984, p.75), cuando afirma que "la suma de dos números impares consecutivos es, por un lado, un múltiplo de 4 y, por otro lado, una diferencia de dos cuadrados: $(p+1)^{2}-(p-1)^{2}=4 p "$. El objetivo de este tipo de problemas es utilizar el lenguaje algebraico para formular problemas en su generalidad y luego resolverlos de manera sistemática. En ese sentido, no existe una superioridad del álgebra sobre la aritmética, sino una dialéctica.

Desde el trabajo de Chevallard desarrollado en 1989, se plantea que el álgebra debe ser considerada una herramienta que permite la construcción de modelos que producen conocimientos acerca del sistema estudiado, a partir del trabajo con parámetros en el estudio de soluciones generales.

Grugeon (2000), apoyada en el trabajo de Chevallard (1989), afirma que la competencia algebraica debe ser evaluada a través de la capacidad de modelar o no problemas matemáticos, para los que el álgebra se presenta como una herramienta adaptada que permite construir un modelo matemático de un sistema (establecer un recorte de la realidad por un conjunto de variables pertinentes); construir un conjunto de relaciones entre las variables (modelo) y luego transformar ese modelo para producir conocimientos sobre el sistema estudiado, dando así sentido a los objetos del álgebra y sus representaciones. Añade además que la competencia algebraica se evalúa también por medio de la capacidad para resolver problemas en los marcos algebraico y funcional que conducen a la manipulación formal de expresiones algebraicas, es decir, trabajar los objetos del álgebra como objetos matemáticos como, por ejemplo, la producción de desigualdades, el estudio de signos, el estudio algebraico de funciones, la búsqueda de valores extremos, etc.

En base a estas nociones, Grugeon (2000) presenta una estructura de análisis multidimensional para la competencia algebraica que cuenta con seis componentes: una componente de identificación/ evaluación que llama de tratamiento algebraico y cinco componentes de caracterización: relación aritmética / álgebra; registro de escrituras algebraicas; articulación entre ese registro y otros; función del álgebra en la actividad matemática y racionalidad algebraica. Estas componentes deben permitir un análisis de la enseñanza y también de los aprendizajes. 
La componente tratamiento algebraico (I) permitirá identificar y comparar diferentes tipos de problemas algebraicos, privilegiados por una determinada institución, así como el grado de implicación del álgebra empleada. Para esta componente se presentan nueve tipos de tratamiento algebraico que son parcialmente jerárquicos, mostrados en la tabla i y numerados por nosotros, que cubren diferentes usos del álgebra.

\begin{tabular}{|c|c|}
\hline Tipos de tratamiento algebraico & Tipos de uso \\
\hline $\begin{array}{l}\text { 1) Realización de tareas que involucran números: } \\
\text { efectuar cálculos o sustituir números en una fórmula. }\end{array}$ & Hacer cálculos numéricos en un contexto de aplicación \\
\hline $\begin{array}{l}\text { 2) Reproducción de tareas formales no finalizadas } \\
\text { - Nivel } 1 \text { (resolución de algoritmos simples) } \\
\text { - Nivel } 2 \text { (cálculos con restricciones, para la } \\
\text { selección de una fórmula adecuada al tratamiento } \\
\text { algebraico que requiere una tarea determinada, } \\
\text { siguiendo un razonamiento que debe ser controlado) }\end{array}$ & $\begin{array}{l}\text { Hacer el cálculo formal en el marco algebraico } \\
\text { - Desarrollar, factorizar, resolver una ecuación de primer } \\
\text { grado, etc. } \\
\text { - Resolver una ecuación de tercer grado sin factor común } \\
\text { aparente, etc. }\end{array}$ \\
\hline $\begin{array}{l}\text { 3) Interpretación de una expresión algebraica que exige } \\
\text { la articulación con un marco o contexto }\end{array}$ & $\begin{array}{l}\text { Asociar dos escrituras de una misma expresión en } \\
\text { registros de representación distintos. }\end{array}$ \\
\hline $\begin{array}{l}\text { 4) Utilización de la herramienta algebraica para } \\
\text { movilizar otras nociones }\end{array}$ & $\begin{array}{l}\text { Estudiar algebraicamente funciones } \\
\text { Estudiar el signo de una expresión algebraica }\end{array}$ \\
\hline $\begin{array}{l}\text { 5) Utilización de herramienta algebraica para modelar } \\
\text { una situación intra o extramatemática en forma de } \\
\text { ecuación }\end{array}$ & $\begin{array}{l}\text { - Resolver problemas en un contexto de aplicación o en } \\
\text { contexto matemático }\end{array}$ \\
\hline $\begin{array}{l}\text { 6) Utilización de la herramienta algebraica para } \\
\text { producir una fórmula o expresar una relación entre } \\
\text { variables }\end{array}$ & $\begin{array}{l}\text { - Matematizar y resolver situaciones intra matemáticas } \\
\text { en otros marcos diferentes del algebraico (geométrico, } \\
\text { numérico, etc.). }\end{array}$ \\
\hline 7) Uso de la herramienta algebraica para generalizar & Modelización de una situación \\
\hline $\begin{array}{l}\text { 8) Utilización de la herramienta algebraica como } \\
\text { herramienta de prueba }\end{array}$ & Expresar una propiedad numérica general \\
\hline
\end{tabular}

Cuadro 1: Tipos de tratamiento algebraico asociados a los usos del álgebra Fuente: Basado en Grugeon (2000, p. 10)

Los diversos tipos de tratamiento algebraico son considerados por la autora como criterios de análisis para esa componente y como un criterio global de análisis, considera el verificar si ellos son privilegiados o no en la enseñanza.

Las otras cinco componentes tienen la función de identificar y describir características importantes y coherencias locales en el currículo oficial para álgebra. La primera de ellas trata de la relación entre aritmética y álgebra (II) con el objetivo de identificar si una determinada enseñanza permite o no una ruptura con la aritmética, a partir de la identificación de la forma de resolución esperada, de la manera como se articulan aritmética y álgebra y, también, de qué objetos del álgebra son necesarios y con qué estatus. Por ejemplo, presenta el enunciado: "cuando 5 se añade a 2 veces un cierto número, la suma es 35" y afirma que la solución aritmética, se hace por un discurso: restar 5 de 35 y luego dividir el resultado por 2. Mientras 
que en una solución algebraica, se tiene que encontrar un número $x$ que verifique la relación $2 x+5=35$, es decir, la expresión indica una adición y una multiplicación, operaciones inversas de la substracción y división de la solución aritmética. Por otro lado, es necesario verificar si este problema es pertinente para justificar la introducción del álgebra. En la tabla ii se presentan los criterios que la autora asocia a esa componente y su posible valor global determinado a priori.

\begin{tabular}{|c|l|l|}
\hline Componentes & \multicolumn{1}{|c|}{ Criterios } & \multicolumn{1}{c|}{ Valores globales } \\
\hline \multirow{4}{*}{$\begin{array}{l}\text { Relación } \\
\text { aritmética/álgebra }\end{array}$} & Enfoque de la resolución & Aritmética / álgebra \\
\cline { 2 - 3 } & Estatus del signo de igualdad & Designa un resultado / equivalencia \\
\cline { 2 - 3 } & Estatus de las letras & $\begin{array}{l}\text { Letra objeto / letra evaluada / Incógnita / } \\
\text { Número generalizado / variable }\end{array}$ \\
\cline { 2 - 3 } & Objeto y estatus de los objetos & $\begin{array}{l}\text { Expresión algebraica / fórmula / ecuación / } \\
\text { función }\end{array}$ \\
\hline
\end{tabular}

Cuadro 2: Estructura de análisis de la relación aritmética/álgebra Fuente: Grugeon (2000, p. 11)

Así, para la autora, el análisis de esa relación entre aritmética y álgebra implicaría la verificación de lo que está siendo tratado en las situaciones presentadas, es decir, si el problema es aritmético o algebraico, siendo fundamental comprobar si no se trata sólo de aritmética generalizada. ¿Cuál es el estatus del signo de igualdad? Representa solo el resultado de un cálculo o representa una equivalencia. ¿Cuál es el estatus de las letras movilizadas? Estos pueden ser: la letra puede estar asociada a un valor numérico, la letra se puede omitir, la letra se puede considerar una etiqueta, la letra puede representar un número desconocido, la letra puede representar un número generalizado que puede asumir varios valores como cuando se utiliza en un contexto funcional como variable. Otro aspecto es verificar qué objeto se está estudiando, es decir, si se trata de una expresión algebraica, una fórmula, ecuación o función.

La segunda componente que tiene la función de identificar y describir características importantes y coherencias locales en el currículo oficial para el álgebra trata de la gestión del registro de expresiones algebraicas (III) y la tercera de la articulación entre ese y otros registros (IV), considerando la dimensión semiótica de la actividad algebraica. Estas componentes tienen por objetivo, desde lo institucional, identificar los registros de representación que están involucrados en la actividad, así como las gestiones que son privilegiadas en el registro de escrituras algebraicas o en su articulación con otros registros a partir de los criterios: tipo de formación, tipo de tratamiento, articulación entre dos registros, reglas de conversión, etc. En la tabla iii se presentan los criterios de análisis de esta componente con sus valores globales. 


\begin{tabular}{|l|l|l|}
\hline \multicolumn{1}{|c|}{ Componentes } & \multicolumn{1}{|c|}{ Criterios } & \multicolumn{1}{c|}{ Valores globales } \\
\hline $\begin{array}{l}\text { Gestión en el registro de } \\
\text { expresiones algebraicas }\end{array}$ & $\begin{array}{l}\text { Correcto: / interpretado (relacionado con el } \\
\text { Tipo de formación } \\
\text { Tipo de tratamiento } \\
\text { Correcto / Interpretado (vinculado al problema) / } \\
\text { No interpretado }\end{array}$ \\
\hline $\begin{array}{l}\text { Articulación entre el registro } \\
\text { de expresiones algebraicas y } \\
\text { otros registros }\end{array}$ & Tipo de conversión & $\begin{array}{l}\text { Correcto / Interpretado (relacionado con el } \\
\text { problema) / No interpretado }\end{array}$ \\
\hline
\end{tabular}

Cuadro 3: Estructura de análisis de la componente gestión del registro y articulación Fuente: Grugeon (2000, p. 11)

El tipo de formación y el tipo de tratamiento constituyen criterios de análisis para la componente de gestión del registro de expresiones algebraicas, mientras que el tipo de conversión es un criterio de la componente referida a la articulación entre el registro de expresiones algebraicas y otros registros. Para la autora, es en la identificación de las reglas de formación y de los tratamientos en el registro de expresiones algebraicas, además de las reglas de conversión y los modos de gestión a ellas asociadas, que se pueden identificar las que son privilegiadas en las tareas de cada institución.

En cuanto a las reglas de formación, afirma que las expresiones algebraicas son escritas y construidas alrededor de un símbolo de operación cuyo operador puede ser unitario, como en el caso de potencias, raíces, etc., o binario, como en el caso de sumas, diferencias, productos, cocientes. Pero hay otros casos en los que son implícitos, como el signo de multiplicación en $\mathrm{ab}$, y otros en los que se emplean convenciones, tales como el punto como signo de multiplicación en $x=1 \cdot x$; o también cuando se considera no necesario colocar una expresión entre paréntesis en el numerador o denominador de una fracción, entre otras situaciones.

En cuanto al tratamiento en el registro de expresiones algebraicas, la autora señala que existen algunos subconjuntos de reglas propias como valores globales de ese criterio de análisis. Entre ellos, presenta el conjunto de reglas de transformación de fracciones, de decimales, de desarrollo (productos notables, propiedad distributiva de la multiplicación respecto a la adición), el conjunto de reglas de transformación para resolver una ecuación del primer grado del tipo $a x+b=0$ o en forma de un producto de factores de primer grado; hacer la misma operación en los dos miembros de una igualdad o aplicar la propiedad "si un producto de factores es nulo uno de los factores es nulo".

La autora considera como registros principales el registro de expresiones numéricas, de expresiones algebraicas, de lengua natural, de figuras, de representaciones gráficas, de 
algoritmos, así como los conjuntos de reglas de conversión entre ellos.

Para la autora, la componente función del álgebra (V) tiene el papel de identificar diferentes formas de actividades algebraicas que privilegien o no diferentes empleos de álgebra y técnicas asociadas y que permitan identificar cual es proceso algebraico idóneo para la resolución de un problema. Desde la enseñanza, la autora presenta el criterio del empleo del álgebra con los siguientes valores globales: el cálculo numérico puede ser o no una herramienta, hacer el cálculo formal en el marco algebraico, estudiar algebraicamente funciones, matematizar, resolver situaciones extra matemáticas, resolver problemas de matemáticas, resolver problemas de matemáticas en contexto de aplicación terciaria, matematizar, resolver una situación intra matemática, conjeturar y probar propiedades numéricas. La autora afirma que esta componente permite diferenciar las relaciones que establece la institución respecto de los objetos algebraicos, porque pueden tener enfoques diferentes y pueden ser observados en los programas oficiales, en los cuadernos de los alumnos y en el libro didáctico.

Para esta componente, la autora sugiere la definición de expresiones algebraicas, de ecuaciones, de inecuaciones, de sistemas lineales, de funciones, de la noción de recta, las reglas de cálculo algebraico, las reglas asociadas a la resolución de ecuaciones, de inecuaciones y de sistemas de ecuaciones lineales, así como las propiedades de ecuaciones y de funciones. En estos casos, afirma que las diferencias pueden aparecer en el grado de complejidad de las expresiones, en los diferentes usos y concepciones para las letras; en las concepciones asociadas a los objetos del álgebra; en la interpretación global o local asociada a una noción; en los tipos de tratamiento empleados y en la utilización de la dimensión funcional.

En cuanto al álgebra escolar, afirma que los principales objetos considerados son las fórmulas, el cálculo algebraico elemental, las ecuaciones, las funciones afines, la noción de recta y estos se asocian a tareas como "manipular formalmente una expresión algebraica", "resolver un problema" , "representar por ecuaciones un problema", "justificar una respuesta", "verificar un resultado" entre otras. Por otro lado, la exploración de esos objetos, en cuanto al saber hacer, se caracteriza por los tipos de ejercicios y métodos de resolución asociados, por el lugar que ocupan los ejercicios modelos, por la mayor o menor frecuencia de los ejercicios dados a los alumnos.

La última componente, racionalidad algebraica (VI), tiene el papel de identificar la relación con la racionalidad matemática que está en juego en la actividad algebraica, es decir, determinar cómo se introducen y cómo se utilizan las propiedades generales o fórmulas en la enseñanza. A esta componente se asocian criterios de análisis como tipos de prueba, tipos de 
justificación (argumentación, recurrir al contenido, evocación a ejemplos, recurrir a procedimientos o propiedades generales, inferencias lógicas). Esta componente permite responder a la siguiente pregunta: ¿qué tipo de prueba se espera?

Para ilustrar la complejidad del análisis del desarrollo de la competencia algebraica, se propone elaborar una tabla para cada tarea, como la tabla iv, elaborado por Grugeon (1995) para la tarea: "escribir una ecuación utilizando A y P para representar la siguiente frase "Hay seis veces más alumnos que profesores en este liceo". (Ibid, p.24).

\begin{tabular}{|l|l|l|}
\hline \multicolumn{1}{|c|}{ Componentes de análisis } & \multicolumn{1}{|c|}{ Criterios } & \multicolumn{1}{|c|}{ Valores } \\
\hline Tratamiento algebraico & $\begin{array}{l}\text { Producción de una expresión para } \\
\text { traducir una situación }\end{array}$ & $\mathrm{Si}$ \\
\hline Relación aritmética/álgebra & Estatus de las letras & $\begin{array}{l}\text { Números generalizados } \\
\text { Variables }\end{array}$ \\
\hline $\begin{array}{l}\text { Articulación entre registro } \\
\text { algebraico y otros registros } \\
\text { semióticos }\end{array}$ & Registros & $\begin{array}{l}\text { Registro algebraico (RA) } \\
\text { Registro de lengua natural (RLN) } \\
\text { RA } \leftarrow \rightarrow \text { RLN }\end{array}$ \\
\hline Función del álgbera & Empleo del álgebra & $\begin{array}{l}\text { Formular uma situación intra o } \\
\text { extra matemática }\end{array}$ \\
\hline
\end{tabular}

Cuadro 4: Tabla de análisis para una tarea

Fuente: Grugeon (1995, p.224)

Aunque la autora ha desarrollado su análisis comparando la intención de la institución con la producción de los alumnos, creemos que esos criterios, asociados a los procesos de modelización algebraica y de algebrización permitirán evaluar si el álgebra escolar permite efectivamente el desarrollo de una competencia algebraica, garantizando los procesos de modelización y algebrización. De esta forma, en lo que sigue, presentaremos en qué consiste el proceso de modelización algebraica.

\subsection{El Proceso De Modelización Algebraica}

El proceso de modelización contempla, de acuerdo con Chevallard (1989, p.53), tres etapas:

En la primera, se define el sistema que queremos estudiar, especificando los "aspectos" relevantes en relación al estudio que pretendemos hacer de ese sistema, es decir, el conjunto de variables por las cuales lo destacamos en el campo de la realidad donde aparece. En la segunda, construimos el modelo estableciendo cierto número de relaciones, R, R', R". etc., entre las variables consideradas en la primera etapa, siendo el conjunto de esas relaciones el modelo del sistema a estudiar. En la tercera, trabajamos el modelo así obtenido, con el objetivo de producir conocimientos acerca del sistema estudiado, conocimientos que toman la forma de nuevas relaciones entre las variables del sistema. El paso 3 es siempre una fase propiamente matemática, mientras que las anteriores son del dominio de la realidad a la que pertenece el sistema - la matemática trata de un objeto matemático, etc. (Traducción nuestra). 
Bolea (2002) presenta cuatro etapas para el proceso de modelización algebraica. La primera etapa está dada por el sistema a modelar, es decir, la situación extra o intra matemática que será estudiada y por las cuestiones generales que pueden surgir respecto a ese sistema que, al principio, no posee respuestas inmediatas. La elección del tipo de modelo que se pretende construir implica, por ejemplo, elegir usar diferentes variables o sólo una.

La segunda etapa, que consiste en la construcción del modelo, implica la identificación y designación de las variables pertinentes que lo caracterizan, además del establecimiento de relaciones entre las variables. En esta etapa, las técnicas utilizadas para la construcción del modelo pueden tener complejidad creciente: expresar en lenguaje algebraico una cantidad dada; designar verbalmente una cantidad compuesta por otras que aparecen en el sistema y expresarla en lenguaje algebraico; y designar y expresar algebraicamente una misma cantidad de dos maneras diferentes. Las expresiones algebraicas que aparecen en esta etapa pueden provenir de dos tipos de actividades: una que permite construir un modelo algebraico a partir de un sistema de varias variables o parámetros y otra que permite construir un modelo algebraico a partir de un sistema de datos e incógnitas.

La tercera etapa comprende el trabajo con el modelo, es decir, la manipulación algebraica propiamente, con el objetivo de obtener un modelo final que muestre las propiedades del sistema modelado y la interpretación de ese trabajo y de los resultados encontrados. Este proceso se caracteriza por las técnicas utilizadas para identificar las componentes de una expresión; identificar la estructura de una expresión; aplicar manipulaciones formales, tanto para obtener expresiones equivalentes, como para expresar una cantidad en función de otras. A su vez, este trabajo con el modelo puede ser subdividido en dos subtipos e incluir tanto la manipulación y la transformación de expresiones, como su interpretación.

Para Bolea (2002), el primer subtipo se refiere al trabajo con variables o parámetros para manipular expresiones algebraicas con distintas variables, como por ejemplo, pedir que desarrollen $(2 x+3 y)^{2}$ tanto por aplicación directa de la fórmula para un binomio al cuadrado, como por la propiedad distributiva, o pedir que expresen la medida del área de un rectángulo que tiene por lados segmentos de longitud $x+4$ y $y+6$ unidades. También consiste en sustituir o desarrollar una fórmula con más de una variable o parámetro proveniente del trabajo de modelización como, por ejemplo: si $A=\frac{a . b}{2}$, ¿cuánto vale $b$ si $A=140$ y $a=20$ ? O si la medida del área de un rombo es de $140 \mathrm{~m}^{2}$ y la diagonal más grande mide $20 \mathrm{~m}$, ¿cuánto medirá la diagonal más pequeña? En ambos casos, dado el modelo, es necesario sustituir los datos y 
luego resolver una ecuación de primer grado con técnicas ya estudiadas.

En ese primer subtipo podemos interpretar expresiones algebraicas con varias variables o parámetros como, por ejemplo: lo que puede representar $x+5=3(y+5)$ que, aunque puede ser similar a los casos anteriores, también se pueden interpretar las operaciones como si provinieran de la siguiente expresión "dentro de cinco años la edad del padre será el triple de la edad del hijo".

El segundo subtipo abarca el trabajo con datos e incógnitas para sustituir o desarrollar en modelizaciones dadas como comprobar que $2 n+1$ es impar para $n=3, n=5, n=2$, o también encontrar el valor de $x$ para que $\frac{8}{12}=\frac{12}{x}$ sea una proporción. Podría incluir también la manipulación de expresiones algebraicas y la resolución de ecuaciones con datos e incógnitas como, por ejemplo, resolver $42+x=3(10+x)$. O interpretar fórmulas con una variable o incógnita como: ¿qué representa $2(n+1)$ ? También reemplazar en fórmulas dadas con datos e incógnitas como: calcular el valor de $A=x^{3}+15 x^{2}-18 x+3 \operatorname{para} x=3, x=0, x=6$. Y, además, la resolución de ecuaciones sin modelización, ni parámetros como: resolver $\frac{5 x-1}{2 x}-$ $\frac{2 x+3}{7}=\frac{3}{x}+x$.

Finalmente, en la cuarta etapa del proceso de modelización, pueden ser enunciados problemas que amplíen el conocimiento del sistema estudiado y la organización de los resultados ya obtenidos. Bolea (2002) afirma que cuanto más algebrizada está una organización matemática, más generales pueden ser tratados los problemas a partir de parámetros; es decir, en esta etapa es fundamental la manipulación de la estructura global de un problema. Otro aspecto a considerar es que la organización matemática debe permitir describir los tipos de problemas de acuerdo con ciertas técnicas a fin de posibilitar la discusión acerca de la solución, si existe o no, si es única o no, etc. Para que estas discusiones sean posibles, la organización matemática en cuestión no puede ser puntual, sino local, pues debe tener un número suficiente de tipos de problemas y de técnicas.

Para Fonseca, Gascón y Lucas (2014, pp. 294-295), hay tres modificaciones importantes en la forma de interpretar la noción de "modelización matemática". La primera trata de incluir la modelización intra matemática en la noción de modelización, como ejemplo, la modelización algebraica de un sistema numérico o geométrico. La segunda es que los modelos construidos en la modelización matemática tienen estructura praxeológica y que la función de los modelos construidos no es la de producir una imagen fidedigna del sistema modelado, es decir, "la modelización matemática presupone la modelización de una praxeología en su totalidad 
mediante otra praxeología matemática". Finalmente, la tercera, trata de interpretar "la modelización matemática como un instrumento capaz de articular y dar funcionalidad a la actividad matemática escolar. La TAD describe los procesos de modelización como procesos de reconstrucción y articulación de organizaciones matemáticas de complejidad creciente.

Las reflexiones vertidas en esta parte del artículo evidencian las etapas del proceso de modelización del álgebra y los aspectos conceptuales en ella involucrados. Los resultados de esas reflexiones nos llevan a pensar en el proceso de algebrización escolar. Desde esta perspectiva, investigadores como Munzón, Bosch y Gascón (2010 (a), 2010 (b), 2015), apoyándose en los estudios de Chevallard (1989), presentan el proceso de algebrización escolar, interpretando, en un primer momento, el álgebra como una herramienta que permite la modelización de todas las áreas de las matemáticas. Es sobre ese proceso de algebrización que trataremos en lo que sigue.

\subsection{Proceso De Algebrización}

Munzón, Bosch y Gascón (2015, p.115) presentan tres etapas para el proceso de algebrización escolar. Para los autores, el punto de partida de tal proceso requiere de un sistema inicial para ser modelado que, en este caso, agrupa programas de cálculo aritmético (PC), es decir, una secuencia de operaciones aritméticas que se puede efectuar "paso a paso". Así, la primera etapa del proceso de algebrización consiste en considerar el programa de cálculo aritmético como un todo y no sólo como un proceso que traduce la reformulación retórica del programa de cálculo para una formulación escrita. Esta etapa no implica necesariamente el uso de letras para representar números; lo importante es la jerarquía de las operaciones y las normas para el uso de paréntesis que hacen surgir un nuevo discurso tecnológico-teórico que incluye la noción de expresiones algebraicas y programas de cálculo equivalentes. Por ejemplo, el problema: "Gabriel pensó en un número, sumó 25 , dividió el resultado por 2 y restó 8 , luego multiplicó el resultado por 3 y obtuvo 21 " puede ser resuelto retóricamente por un discurso: "si al final se obtiene 21, antes de multiplicar por 3 tenía 7, antes de quitar 8 tenía 15 , antes de dividir por 2 tenía 30 y antes de sumar 25 tenía 5. Luego, Gabriel pensó en el número 5. "Esa

formulación retórica se puede traducir al siguiente programa de cálculo: $\left(\frac{21}{3}+8\right) \times 2-25$ que es equivalente a $(7+8) \times 2-25$, y que a su vez es equivalente a $15 \times 2-25$, y luego a $30-$ 25 = 5, el número pensado por Gabriel. (Munzón, Bosch y Gascón, 2010 (a), p.659).

Al ampliar los problemas de esta etapa, los autores consideran aquellos en los que se 
presentan datos numéricos conocidos y lo desconocido es una cantidad, pero que evolucionan hacia problemas en los que no todos los datos son numéricos y la incógnita no es un resultado numérico. Por ejemplo, muestran el siguiente problema: "Noelia y Marga piensan, independientemente, en un número. Noelia multiplica su número por 3 , sustrae 18 y divide el resultado por 9. Marga sustrae 4 del número que pensó, después multiplica por 5 y termina dividiendo el resultado por $10 . \mathrm{Si}$, casualmente, obtiene el mismo resultado final, ¿qué relación hay entre los números pensados por Noelia y Marga? "(Munzón, Bosch y Gascón, 2011, p.749). Afirman que esta etapa puede contemplar aún problemas que conducen a la resolución de ecuaciones del primer grado en los que la incógnita aparece sólo en uno de los miembros. Básicamente, en esta etapa, se desarrollan técnicas de simplificación de expresiones algebraicas.

Para los autores, el paso a la segunda etapa de algebrización ocurre cuando las técnicas de simplificación de un PC no son suficientes para resolver el problema, es decir, cuando los datos del problema y la incógnita aparecen como relaciones entre las variables que se caracterizan por una la igualdad entre dos PCs. En este caso, el cálculo se vuelve más complejo y las ecuaciones se consideran entonces un nuevo objeto matemático que implica nuevas técnicas, como la de cancelación y la obtención de ecuaciones equivalentes pero no programas de cálculo equivalentes, pese a que la solución sigue siendo un valor numérico. Se presentan como ejemplo: "Marta piensa en un número y lo suma al doble de su consecutivo. Si el resultado final es 4 unidades mayor que el doble del número pensado, ¿se puede determinar qué número pensó Marta? (Munzón, Bosch y Gascón, 2010a, pág. 663). En este caso, el primer programa de cálculo puede estar representado por: $P C(n)=n+2(n+1)$ que puede transformarse en el equivalente: $P C_{1}(n)=3 n+2$ y el segundo por: $P C_{2}(n)=2 n+4$. Luego, será necesario transformar en una igualdad y aplicar técnicas de resolución de ecuaciones para obtener el resultado $n=2$. En esta etapa se inicia el cambio del uso del signo de igualdad, pasando ahora a representar la igualdad entre dos programas de cálculo. En el caso de los sistemas de ecuaciones lineales podemos considerar el problema: Diego ahorró 235 reales en monedas de 1 y 50 centavos. Si en total son 340 monedas, ¿cuántas monedas de cada tipo tiene Diego? Por lo tanto, si $n$ representa la cantidad de monedas de 50 centavos, los programas de cálculo $P C_{1}(n)=235-0.5 n$ y $P C_{2}(n)=340-n$ representan la cantidad de monedas de denominación 1.

Para los autores, ese es el modelo dominante del álgebra escolar, es decir, aquel en el que el álgebra permite convertir una representación en el registro del lenguaje natural a otra 
representación en el registro del lenguaje algebraico y luego pasar a la resolución de ecuaciones, generalmente, con una incógnita. Sin embargo, los autores proponen un modelo más amplio considerando relaciones entre programas con varias variables que conducen al próximo paso.

La tercera etapa del proceso de algebrización se presenta cuando no se limita el número de variables y la distinción entre parámetros e incógnitas desaparece, lo que posibilita ampliar los conocimientos del sistema modelado con el estudio de cuestiones problemáticas que no pueden ser discutidas en el sistema. Los programas de cálculo, en esta etapa, pueden ser interpretados como "fórmulas" o modelos algebraicos de un sistema matemático o extramatemático, o sea, a la generalización de problemas de la etapa anterior buscando técnicas para entender cómo cada variable depende de las otras. Un ejemplo de problema de esta etapa, presentado por los autores es: ¿¿Cuál relación existe entre el perímetro $P$ y la medida del área $A$ de un triángulo isósceles? ¿En qué casos $P$ y $A$ determinan un único triángulo isósceles? (Munzón, Bosch y Gascón, 2010b, p. 553). En esta etapa de algebrización escolar, se incluirían entonces las tareas de construcción y análisis de modelos, y entendemos que en ella deben ser consideradas las etapas del proceso de modelización algebraica. Los autores señalan la necesidad de avanzar hacia la modelización funcional.

\section{La Articulación}

En nuestro análisis partiremos de las etapas de los procesos de algebrización y modelización a las cuales asociaremos los tipos de tratamientos presentados en la tabla i, así como los otros componentes de la competencia algebraica.

En la primera etapa del proceso de algebrización, en la componente relación entre aritmética y álgebra, se ubicarían aquellos problemas para los que el tratamiento numérico es suficiente o aquellos en los que el álgebra se concibe como aritmética generalizada. El cálculo algebraico sería una prolongación del cálculo numérico en el que la letra dejaría de ser una etiqueta para asumir el status de incógnita. Aunque la función de álgebra se restringe solo al uso del cálculo numérico como herramienta, se inicia el trabajo con expresiones algebraicas y ecuaciones, a las que les corresponderían sólo justificaciones por propiedades aritméticas en la componente racionalidad algebraica. Se entiende que permanecen los tratamientos en el registro numérico y se inicia la formación del registro algebraico a partir de la traducción de problemas por ecuaciones, lo que configuraría la conversión de representaciones del registro de la lengua natural o figural a representaciones algebraicas. El primer tipo de tratamiento algebraico (1) se 
desarrolla en las tareas que implican efectuar cálculos con números o sustituirlos en una fórmula, articulando el registro algebraico y numérico. En esta etapa, también se inicia el uso de letras para designar cantidades desconocidas en el sentido de utilizar expresiones literales, traducir relaciones y luego resolverlas por medio de ecuaciones resueltas por simplificación.

En la segunda etapa del proceso de algebrización, además del status de incógnita, las letras pueden asumir el papel de número generalizado o variable, apareciendo en los dos miembros de la ecuación, lo que haría que el signo de igualdad de la ecuación pase a representar una relación de equivalencia y no una respuesta. La ecuación se convierte en un objeto de estudio y se deben desarrollar nuevas técnicas, como la cancelación. El estudio efectivo de ecuaciones y fórmulas favorece la introducción de la noción de función y otros recursos de tratamiento, como la factorización.

El paso a esta etapa amplía la gestión del registro algebraico, aunque permanecen las conversiones de representaciones de los registros de la lengua natural y figural para representaciones del registro algebraico. La función del álgebra pasa a contemplar también el cálculo formal, la resolución de problemas intra y extra matemáticos, la producción de fórmulas simples, además del trabajo con propiedades algebraicas que justifican los nuevos tratamientos (2), (3), (6), y (4), de acuerdo con la tabla 1.

El segundo tipo de tratamiento algebraico (2), de acuerdo con Grugeon (1995), se refiere al tratamiento formal de expresiones algebraicas que ocurre en dos niveles. En el primero, este se produce a partir de un trabajo algebraico de aplicación del saber hacer básico, como por ejemplo, la factorización o el desarrollo de expresiones y la resolución directa de ecuaciones de primer y de segundo grado. En el nivel 2 de este tipo de tratamiento, la manipulación formal exige el cálculo algebraico con restricciones que implican la selección del tratamiento más adecuado en tareas más complejas de factorización, de transformación de fórmulas, etc. La autora presenta como ejemplo, "factorizar la expresión $(x+1)^{3}+1-x^{2}$ o resolver la ecuación $4 x^{3}-x=0$ o también si $y=-2 x$ y $z=y^{2}-3 y$, exprese $z$ en función de $x$. (Grugeon, 1995, p. 52).

El tercer tipo de tratamiento algebraico (3) implica la interpretación de una expresión algebraica asociada a un marco o a un contexto y permite el trabajo de interpretación en situaciones de reconocimiento de expresiones tanto en el marco algebraico, como en otros marcos, como por ejemplo, identificar entre varias expresiones aquella que es equivalente a la expresión $(x+2)(x-3)(x+4)$, donde $x$ representa un número real cualquiera.

El cuarto tipo de tratamiento (4) permite la "utilización de la herramienta algebraica para 
hacer funcionar otras nociones matemáticas". En el estudio de esas nociones, las herramientas algebraicas serían movilizadas para tareas efectivamente algebraicas, principalmente, en el marco funcional. Por ejemplo, "mostrar que, para todos los números reales $x, x^{2}-3 x+10>$ 0. (Grugeon, 1995, p. 54).

En la segunda etapa del proceso de algebrización, el quinto tipo de tratamiento algebraico (5), se refiere a la utilización de la herramienta algebraica para modelizar, en forma de ecuación, una fase intra o extra matemática. Las tareas requerirían la producción de una expresión o relación algebraica que traduciría algebraicamente una situación como la siguiente: "si el lado de un rectángulo tiene una longitud de $1 \mathrm{~cm}$, determine la longitud del otro lado para que tal rectángulo posea una razón de proporcionalidad entre sus lados igual a la razón áurea".

En el tipo de tratamiento (6), la utilización de la herramienta algebraica consiste en producir fórmulas más complejas o expresar una relación entre variables, pudiendo englobar la traducción directa o la traducción a partir de la aplicación de un teorema (generalmente de contextos de geometría en donde se obtiene una relación entre los datos y las incógnitas). Otros problemas pueden implicar propiedades matemáticas sobre números o en relación a la escritura genérica de números. Como ejemplo de este tipo de tratamiento, la autora presenta el siguiente problema: "En una tienda se venden camisas y pantalones. Todas las camisas tienen el mismo precio unitario. Todos los pantalones tienen el mismo precio unitario. Juan pagó 570 reales por 7 camisas y 3 pantalones. Sofía pagó 730 reales por 3 camisas y 7 pantalones. Calcular el precio de una camisa y de un pantalón." Otro ejemplo: " Un padre tiene dos veces la edad de su hijo. En quince años, la edad del padre será el doble de la edad de su hijo. ¿Cuáles son las edades respectivas del padre y del hijo? "(Grugeon, 1995, p.54).

Estos tipos de tratamientos, desarrollados en la segunda etapa del proceso de algebrización, a partir de relaciones entre incógnitas y los datos del problema, podrían ser situados también en la primera etapa del proceso de modelización algebraica, porque se propone un modelo para solución de sistemas de ecuaciones lineales, ecuaciones o inecuaciones. Así, podemos decir que en este segundo nivel del proceso de algebrización, estamos desarrollando conocimientos que deberán ser movilizados en el proceso de modelización.

En la tercera etapa del proceso de algebrización, las letras siguen representando incógnitas, números generalizados o variables. En cuanto a los registros, se puede introducir la representación gráfica y, por lo tanto, se contempla la conversión de una representación del registro algebraico a una representación en el registro gráfico y viceversa. La función del álgebra en esta etapa sería la de proponer el cálculo formal en el marco algebraico, es decir, el 
desarrollo de técnicas, a partir de situaciones extra e intra matemáticas, además de verificar conjeturas y proponer justificaciones.

El tratamiento algebraico del tipo (7), que se refiere a la utilización de la herramienta algebraica para generalizaciones, es ejemplificado por la autora a través del problema: "encontrar tres enteros consecutivos cuya suma sea igual a 54" (Grugeon, 1995, p. 55). En este tipo de tratamiento, podríamos añadir la generalización de problemas, en el sentido de Chevallard (1989), para la búsqueda de fórmulas que resuelven un campo de problemas. En este último ejemplo, podríamos sustituir el número 54 por un parámetro $a$, que permitiría pasar de un simple cálculo a encontrar el valor de $x$ en $x+x+1+x+2=a$ y proponer otras cuestiones como qué característica debe tener el número a para que el problema tenga solución. En este caso, el número a deberá ser múltiplo de 3.

El octavo tipo de tratamiento algebraico (8) que implica la "utilización de la herramienta algebraica como herramienta de prueba" será movilizado en situaciones de contexto matemático que conducen a probar alguna propiedad numérica o geométrica, como por ejemplo: "Sean tres números consecutivos, expresar algebraicamente la diferencia entre el cuadrado del segundo número y el producto del primero y del tercero. ¿Qué puede constatar? Justifique su respuesta. (Grugeon, 1995, p.56).

En esta etapa del proceso de algebrización, se desarrollaría el proceso de modelización, pues esos tratamientos podrían ser movilizados para ampliar el conocimiento del sistema estudiado, para producir pruebas de propiedades, además de justificar soluciones. Sin embargo, nada impide que, para iniciar el estudio de un sistema, se presenten problemas que se encuadren en la primera y en la segunda etapa del proceso de algebrización. Así, a partir del análisis realizado, se puede verificar si la propuesta considerada para la enseñanza del álgebra en una determinada institución promueve el desarrollo de la competencia algebraica, satisfaciendo los procesos de algebrización y modelización.

Para sintetizar la articulación elaborada, en esta sección del artículo, presentamos la tabla v. Partimos de las etapas del proceso de algebrización, a las cuales, según las relaciones identificadas, asociamos convenientemente las etapas del proceso de modelización y, a continuación, los tipos de tratamiento, así como las componentes para el desarrollo de la competencia algebraica.

Creemos que es posible organizar la tabla $v$ para cada tema de enseñanza del álgebra escolar, identificando en cada etapa la organización matemática local necesaria. Luego, para cada una de esas organizaciones matemáticas se puede desarrollar un cuadro, como el cuadro 
5, que especifique lo que favorece o no el desarrollo de la competencia algebraica.

\begin{tabular}{|c|c|c|c|c|c|}
\hline Algebrización & Modelización & $\begin{array}{c}\text { I } \\
\text { tipos de } \\
\text { tratamiento }\end{array}$ & \begin{tabular}{|c|} 
II \\
aritmética/álgebra
\end{tabular} & $\begin{array}{l}\text { III y IV } \\
\text { gestión de los } \\
\text { registros y } \\
\text { conversión }\end{array}$ & $\begin{array}{c}\text { V y VI } \\
\text { función del } \\
\text { álgebra y } \\
\text { racionalidad } \\
\text { algebraica } \\
\end{array}$ \\
\hline $\begin{array}{c}1^{\mathrm{a}} \text { Etapa - PC } \\
\text { como un todo. } \\
\text { Pueden o no } \\
\text { aparecer letras. El } \\
\text { foco son las reglas } \\
\text { para las } \\
\text { expresiones } \\
\text { numéricas }\end{array}$ & \begin{tabular}{|c|} 
Construcción de \\
conocimientos que \\
serán movilizados \\
durante el proceso \\
de modelización
\end{tabular} & 1 & $\begin{array}{c}\text { Aritmética } \\
\text { generalizada. } \\
\text { Álgebra. }\end{array}$ & $\begin{array}{c}\text { Registro de la } \\
\text { lengua natural } \\
\text { (RLN). } \\
\text { Registro numérico } \\
\text { (RN). } \\
\text { Registros de } \\
\text { expresiones } \\
\text { algébricas (REA). } \\
\text { RLN } \leftrightarrow \text { REA } \\
\end{array}$ & \begin{tabular}{|c|} 
Cálculo \\
aritmético como \\
herramienta. \\
Justificaciones a \\
través de \\
propiedades \\
aritméticas.
\end{tabular} \\
\hline $\begin{array}{c}2^{\text {a }} \text { Etapa - La letra } \\
\text { como número } \\
\text { generalizado o } \\
\text { variable. } \\
\text { Ecuaciones como } \\
\text { objeto. Técnicas } \\
\text { de resolución. Se } \\
\text { consideran varias } \\
\text { variables. }\end{array}$ & \begin{tabular}{|c|} 
Construcción de \\
conocimientos que \\
serán movilizados \\
durante el proceso \\
de modelización.
\end{tabular} & $\begin{array}{l}2 \\
3 \\
6 \\
4\end{array}$ & $\begin{array}{c}\text { Álgebra. } \\
\text { Ecuación como } \\
\text { objeto de estudio. } \\
\text { Interpretación de } \\
\text { expresiones } \\
\text { Producción de } \\
\text { fórmulas simples }\end{array}$ & $\begin{array}{c}\text { Registros anteriores } \\
\text { y Registro figural } \\
\text { (RF). } \\
\text { RLN } \leftrightarrow \text { REA } \\
\text { RF } \leftrightarrow \text { REA }\end{array}$ & \begin{tabular}{|c|} 
Cálculo formal en \\
problemas intra y \\
extra matemáticos \\
Propiedades \\
algebraicas que \\
justifican nuevos \\
tratamientos
\end{tabular} \\
\hline \multirow{3}{*}{$\begin{array}{c}3^{\text {a }} \text { Etapa }- \\
\text { Desaparece la } \\
\text { distinción entre } \\
\text { parámetros e } \\
\text { incógnitas. } \\
\text { Fórmulas o } \\
\text { modelos } \\
\text { algebraicos del } \\
\text { sistema } \\
\text { matemático o } \\
\text { extra matemático. }\end{array}$} & \multirow{3}{*}{\begin{tabular}{|c|}
$1^{\text {a }}$ Etapa - Sistema \\
a modelar. \\
Situación \\
matemática o extra \\
matemática que \\
será estudiada a \\
través de preguntas \\
generales. \\
$2^{\mathrm{a}}$ Etapa - \\
construcción del \\
modelo con \\
identificación y \\
designación de \\
variables y \\
establecimiento de \\
establecimiento de \\
relaciones entre \\
ellas. \\
$3^{\text {a Etapa }-}$ \\
Obtención del \\
modelo final que \\
muestra las \\
propiedades del \\
sistema \\
modelizado
\end{tabular}} & $\begin{array}{l}3 \\
5 \\
6\end{array}$ & \begin{tabular}{|c|} 
Álgebra. \\
Modelo para \\
solución (ecuaciones \\
o sistemas de \\
ecuaciones lineales, \\
inecuaciones) \\
Relaciones entre \\
incógnitas y datos. \\
\end{tabular} & $\begin{array}{c}\text { Registros anteriores } \\
\text { y Registro Gráfico } \\
\text { (RG) } \\
\text { REA } \leftrightarrow \text { RG }\end{array}$ & \begin{tabular}{|c|} 
Cálculo formal en \\
el marco \\
algebraico \\
Elaboración de \\
conjeturas y \\
justificaciones.
\end{tabular} \\
\hline & & $\begin{array}{l}4 \\
5 \\
6\end{array}$ & \begin{tabular}{|c|} 
Álgebra. \\
Regla de tres simple \\
y compuesta. \\
Función afín y \\
cuadrática. \\
Inecuaciones. \\
\end{tabular} & $\begin{array}{c}\text { Todos los registros } \\
\text { y conversiones } \\
\text { anteriores }\end{array}$ & $\begin{array}{l}\text { Álgebra para } \\
\text { producir una } \\
\text { fórmula o } \\
\text { expresar una } \\
\text { relación entre } \\
\text { variables. }\end{array}$ \\
\hline & & $\begin{array}{l}7 \\
8\end{array}$ & \begin{tabular}{|} 
Generalización de \\
clase de problemas. \\
Justificación y \\
prueba de \\
propiedades.
\end{tabular} & $\begin{array}{c}\text { Todos los registros } \\
\text { y conversiones } \\
\text { anteriores }\end{array}$ & $\begin{array}{c}\text { Álgebra como } \\
\text { herramienta de } \\
\text { generalización y } \\
\text { de prueba }\end{array}$ \\
\hline
\end{tabular}

Cuadro 5: Articulación entre algebrización, modelización y componentes de la competencia algebraica

Fuente: De los autores

\section{Consideraciones Finales}

Munzón, Bosch y Gascón (2015) afirman que es desproporcionada la cantidad de 
ejercicios de cálculo algebraico en relación a los que serán exigidos en la resolución de ecuaciones, ya que estos últimos son considerados más simples. Sin embargo, no son suficientes para justificar la importancia dada al cálculo algebraico en la enseñanza secundaria, pues el aprendizaje del cálculo algebraico, en ese nivel de enseñanza, se hace de manera formal y no funcional, el alumno no aprende a "desarrollar", "factorizar " y "simplificar "como medio para resolver o facilitar alguna tarea" (p. 111).

En ese sentido, el considerar al álgebra como proceso de algebrización contribuirá a entender efectivamente el status y la razón de ser del álgebra elemental, pues permitirá abordar cuestiones teóricas de aritmética y geometría, como por ejemplo, el estudio de relaciones, independientemente de la naturaleza de los objetos relacionados. Así, se debe trabajar con soluciones generalizadas para ciertos tipos de problemas, en lugar de resolverlos aisladamente. Esto permitirá también identificar cuáles son las condiciones que harán que los problemas tengan solución.

Las consideraciones anteriores pueden servir como base teórica para el diseño de procesos de formación continua con profesores. En particular, permitirá comprobar que la búsqueda de soluciones generales no es una práctica que se desarrolle en la enseñanza básica. Para ejemplificar este hecho, citamos un problema clásico de la enseñanza de sistemas de ecuaciones lineales, el problema de las gallinas y conejos, que fue trabajado en la formación de profesores, tanto en São Paulo, como en Lima (Perú). El enunciado es el siguiente: En un patio existen gallinas y conejos: en total hay 26 cabezas y 70 patas. ¿Cuántos son gallinas y cuántos son conejos? A continuación se presentó una serie de problemas similares.

Los profesores representaron los datos de los problemas a través de sistemas de ecuaciones lineales, como traducción directa del enunciado al registro de expresiones algebraicas; ninguno tradujo los enunciados a un sistema de ecuaciones general. Luego resolvieron uno a uno los problemas, para lo cual emplearon métodos que solían utilizar. Por ejemplo, tradujeron el enunciado en una sola ecuación: si $g+c=26$ y $2 g+4 c=70$ entonces $4 g+4(26-g)=70$ donde $g$ representa la cantidad de gallinas y $c$ la de conejos.

De otro lado, en la experimentación realizada se han identificado algunas técnicas de solución distintas. Por ejemplo, movilizando apenas conocimientos aritméticos, una de las profesoras del grupo de São Paulo, con experiencia en la enseñanza de la matemática en los años iniciales, resolvió el problema en el marco aritmético, asociando la cantidad de gallinas y conejos con la respectiva cantidad de patas. Para ese tipo de problema, los profesores de Lima presentaron como técnica de solución el método que denominan método del rombo, por la 
forma en la que disponen los datos en el plano. En la figura 1 se presenta el esquema empleado, donde $\mathrm{T}_{1}$ representa la suma de los valores de las incógnitas (en el ejemplo será 26), $\mathrm{T}_{2}$ representa la suma de los valores unitarios de las características asociadas a las incógnitas (en el ejemplo sería el total de patas, 70) representa el valor unitario mayor (en este caso sería 4) y representa el valor unitario menor (que sería 2). Por lo tanto, el valor de la primera incógnita (número de gallinas) se obtiene realizando las siguientes operaciones: $g=\frac{T_{1} a-T_{2}}{b}=\frac{26(4)-70}{2}=$ 17, es decir, la cantidad de gallinas es 17 y, por lo tanto, la operación $26-17=9$ representa la cantidad de conejos.

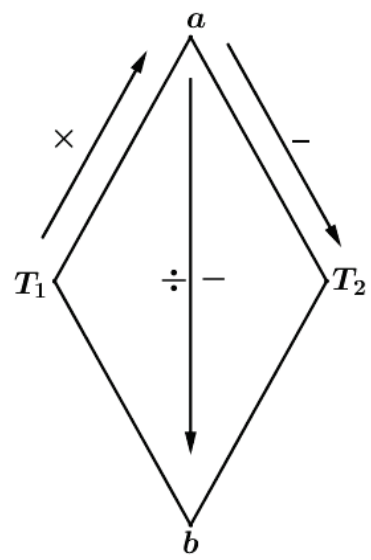

Figura 1 - Método del rombo Fuente: De los autores

De otro lado, es interesante notar que, aunque los profesores contaban con una técnica para el tipo de tarea que relaciona el número de animales y el número de patas, no pudieron justificarla, es decir, no presentaron un discurso tecnológico teórico que la sustentara. Lo mismo sucedió con las técnicas movilizadas por los profesores en São Paulo.

Además, en São Paulo se sugirió a los profesores que organizaran los problemas presentados sustituyendo los datos por parámetros. Optaron por considerar cuatro categorías: problemas del tipo: $g+c=n$ y $2 g+4 c=m$; problemas de tipo $c=3 g$ y $2 g+4 c=n$; problemas del tipo: $g=$ $3 c$ y $2 g+4 c=n$ y problemas del tipo: $v+b=n$ y $v-2 b=m$ en donde $v$ representa la cantidad de vacas y $b$ la cantidad de bueyes. Se plantearon algunas preguntas: ¿qué discusiones pueden favorecer el uso de parámetros en estos problemas? ¿Hay algún modelo para cada categoría creada?

A partir de ello, los profesores se centraron en el problema de la categoría 1 y presentaron las ecuaciones de la siguiente forma: $g+c=y$ y $2 g+4 c=x$, donde las letras $x$ e y fueron empleadas como parámetros. Emplearon el método de adición para resolverlo, obteniendo que $x-2 y=2 c$ o $c=\frac{x-2 y}{2}$ y que $4 y-x=2 g$ o $g=\frac{-x+4 y}{2}$, sin observar las 
condiciones necesarias que debían satisfacer los valores de $x$ e $y$. Luego abordaron el problema de la categoría 4 y plantearon las ecuaciones $v+b=x$ y $v-2 b=y$; por el mismo proceso usado anteriormente, concluyeron que $x-y=3 c$ o $b=\frac{x-y}{3}$ y también que $2 x+y=3 v$ o $v=\frac{2 x+y}{3}$. Señalaron que la diferencia entre los dos parámetros debía ser un múltiplo de $3 \mathrm{y}$, a continuación, retomaron los problemas para identificar los valores de $x$ e $y$ y verificar si realmente el modelo encontrado era solución del problema. Sin embargo, para ello consideraron exactamente los números inicialmente en el enunciado; no recurrieron a otros valores.

Pese al trabajo realizado en las formaciones desarrolladas, tanto en Lima como en Sao Paulo, los profesores no progresaron más en la discusión ya que no percibieron el sentido del trabajo con parámetros. Así por ejemplo, no reconocieron que un cambio en los valores del parámetro originaba que el problema tuviera solución, o que no la tuviera o que estas fueran infinitas. Entendemos que eso se debe a que su práctica habitual consiste en resolver sistemas y no en discutir cuáles son las condiciones bajo las cuales estos tienen solución. De otro lado, también se encontró que difícilmente volvían al contexto del problema para interpretar la solución encontrada.

Luego de desarrollar procesos de formación tanto en Lima como en Sao Paulo centrado en problemas que requieren del empleo de sistemas de ecuaciones lineales, se encontró que los profesores no perciben el sentido del trabajo con parámetros. Así por ejemplo, no reconocen que un cambio en los valores del parámetro originará que el problema tenga solución, o que no la tenga, que estas sean infinitas. Entendemos que eso se debe a que su práctica habitual consiste en resolver sistemas y no en discutir cuáles son las condiciones bajo las cuales estos tienen solución. De otro lado, también se encontró que difícilmente retornan al contexto del problema para interpretar la solución encontrada.

Podemos inferir que la enseñanza de sistemas de ecuaciones lineales atiende parte de lo que se describe en las líneas 1 y 2 de la tabla v. El proceso de algebrización se sitúa en la segunda etapa, pues la enseñanza privilegia apenas el cálculo formal y algunas propiedades que justifican nuevos tratamientos o técnicas. No se produce la tercera etapa del proceso de algebrización y, consecuentemente, no se distingue entre parámetros e incógnitas, ni se producen fórmulas ni modelos algebraicos, ya que tampoco se desarrolla el proceso de modelización.

Los problemas resueltos por sistemas de ecuaciones lineales presentan datos que conducen siempre a una única solución única, pues el objetivo es solamente aplicar las técnicas de resolución. No se trabaja con problemas que contengan informaciones redundantes o 
contradictorias de modo que conduzcan, como señala Coulange (2000), a problemas en los que el sistema tiene una solución que no satisface el problema ni a problemas cuyo sistema tiene infinitas soluciones pero que, por el contexto del problema, estas quedan reducidas a los valores que toman en un determinado intervalo. Consideramos que el trabajo de generalización de problemas, empleando parámetros, puede ayudar en la elección de datos que conduzcan a este tipo de situaciones.

Sin embargo, el problema no está solamente en la formación de profesores; de los resultados obtenidos por Fonseca, Gascón y Lucas (2014, p. 293):

hasta el momento muestran que la integración de la modelización matemática en cualquiera de los niveles del sistema educativo choca con restricciones institucionales que van más allá de la voluntad y la formación de los sujetos de las instituciones involucradas, por ejemplo, las que resultan del aplicacionismo y del contrato didáctico vigente.

Añaden que para que la modelización forme parte de los sistemas de enseñanza es necesario construir dispositivos didácticos que permitan superar las dificultades para acoger tal actividad que presentan las propias instituciones antes que las de los sujetos. Retomando a Chevallard (1989), se sugieren cambios a partir del currículo para que la funcionalidad del cálculo algebraico surja mediante producción y exploración de fórmulas, así como de la noción de función. Esos temas, además de la generalización de problemas, deben aparecer como objetivos de enseñanza, de lo contrario, continuaremos tratando el álgebra sólo como productora de herramientas y no como poseedora de diversos objetos de estudio. La inspiración para tales cambios puede estar en libros antiguos, anteriores a las reformas de la Matemática Moderna.

\section{Referências}

Almoloud, S.A. (2007). Fundamentos da Didática da Matemática. Curitiba: Editora da UFP.

Bolea, P., Bosch, M. y Gascón, J. (1998). The role of algebraization in the study of a mathematical organization. In I. Schwank (Ed.), Proceedings of the First Conference of the European Society for Research in Mathematics Education (Vol. II, pp. 135-145). Osnabrück, Germany: CERME.

Bolea, P., Bosch, M. y Gascón, J. (2001). La transposición didáctica de organizaciones matemáticas en proceso de algebrización: el caso de la proporcionalidad. Recherches en Didactique des Mathématiques, 21(3), 247-304.

Bolea, P. (2002). El processo de algebrización de organizaciones matemáticas escolares. Tesis de doctorado, Universidad de Zaragoza, España.

Chevallard, Y. (1984) Le passage de l'arithmetique a l'algebrique dans l'ensegnement des mathematiques au college. Premiere partie, $1^{\prime}$ evolution de la transposition didactique. Petit $x, \mathrm{n} .5, \mathrm{p}$. 51-94. 
Chevallard. Y. (1989) Le passage de l'arithmetique a l'algebrique dasn l'ensegnement des mathematiques au college. Deuxeieme partie, perspectives curriculares : la notion de modelisation. Petit x, n. 19, p. 43-72.

Chevallard, Y. (1990). Lepassage de l'arithmetique a l'algebrique dans l'enseignement des mathematiques au college. Troisième partie. Voies d'attaque et problemas didactiques. Petit $x, \mathrm{n} 23, \mathrm{p}$. $5-38$.

Chevallard, Y. (1992). Concepts fondamentaux de la Didactique: perspectives apportées par une approche anthropologique. Recherches en Didactique des Mathématiques, 12 (1), 73-112.

Chevallard, Y. (1999). L'analyse des pratiques enseignantes en Théorie Anthropologique du didactique. Recherches en Didactique des Mathématiques, 19(2).221-266.

Chevallard, Y. (2002). Organiser 1'etude. 1. Structures \& Fonctions. Actes de la 11 École d'Été de Didactique des Mathématiques. France: La Pensée Sauvage.

Coulange, L. (2000). Étude des pratiques du professeur du double point de vue écologique et économique. Cas de l'enseignement des systèmes d'équations et de la mise en équations en classe de troisième. Tesis de doctorado. Universidad Joseph Fourrier, Grenoble I.

Douady, R. (1986). Jeux de cadre et dialectique outil-objet. Recherche en didactique des Mathématiques. Grenoble: La Pensée Sauvage Éditions, 7(2), 5-31.

Duval, R. (1995). Sémiosis et pensée humaine. Suíça: Peter Lang S.A.

Duval, R. (1999). L'analyse cognitive du fonctionnement de la pensée et de l'activité mathématique. Cours sur les apprentissages intellectuels donné à la PUC-SP. São Paulo : Programa de Estudos PósGraduados em Educação Matemática.

Fonseca, C., Gascón, J. y Lucas, C.O. (2014). Desarrollo de un modelo epistemológico de referencia en torno a la modelización funcional. Revista Latinoamericana de Investigación en Matemática Educativa, 17(3), 289-318.

Gascón, J. (1993). Desarrollo del conocimiento matemático y análisis didáctico. Del patrón análisissíntesis a la génesis del lenguaje algebraico. Recherches en didactique des mathématiques. 13(3), 295332.

Gascón, J. (1994). Un nouveau modele de l'algèbre élémentaire comme alternative à l'arithmétique généralisée".Petit $x$, n. 37, 43-63.

Gascón, J. (1999). La naturaleza prealgebraica de la matemática escolar. Educación Matemática, 11(1), 77-88.

Grugeon, B.(1995). Etude des rapports institutionnels et des rapports personnels des élèves à l'algèbre élémentaire dans la transition entre deux cycles d'enseignement: BEP et Première G. Tesis de doctorado, Universidad Paris VII.

Grugeon, B. (2000) Une structure d'analyse multidimensionnel en algebre elementaire: conception, exploitation et perspectives. Actes de journées de formation de formateurs. IREM, Universidad de Montpellier II.

Munzón, N., Bosch, M. y Gascón, J. (2010a) La algebrización de los programas de cálculo aritmético y la introducción del álgebra en secundaria. En A. Bronner, M. Larguier, M. Artaud, M. Bosch, Y. 
Chevallard, G. Cirade y C. Ladage. (Eds) Diffuser les mathématiques (et les autres savoirs) comme outils de connaissance et d'action. pp. 655-676. II e Congrès international sur la TAD

- (2010b) La algebrización de los programas de cálculo aritmético y la introcución del álgebra em secundaria. In: M.M.Moreno et al (Eds.), Investigación em Educación Matemática XIV, p.545556, Lleida: SEIEM.

- (2011) Um modelo epistemológico de referencia del álgebra como instrumento de modelización. In: M.Bosch et al (Eds), Un panorama de la TAD (vol. 10, p. 743-765). Barcelona, España: Centre de Recerca Matemática.

(2015) El problema didáctico del algebra elemental: Un análisis macro-ecológico desde la teoría antropológica de lo didáctico. REDIMAT, Vol 4(2), p. 106-131.

Sangiorgi, O. (1959) Matemática para a segunda série ginasial. São Paulo: Companhia Editora Nacional. 